\title{
Correlation Analysis of Neuropathic Headache and Depression and Anxiety
}

\author{
Changxin Dou* \\ Department of Neurology, Yidu Central Hospital of Weifang, Weifang 262500, China
}

*Corresponding author: Changxin Dou, douchangxin212@163.com

\begin{abstract}
Objective: To explore the relationship between neuropathic headache, and depression and anxiety, as well as to adopt reasonable methods to reduce patients' depression and anxiety. Methods: From June 2019 to May 2021, a total of 89 neuropathic headache patients were treated in our hospital, which is Yidu Central Hospital of Weifang and they were recorded as the observation group. During the same period, healthy individuals were randomly selected using a random number table method and 91 cases were randomly selected and recorded as the control group. Through the questionnaire survey and the use of SAS and SDS scores, the anxiety and depression of healthy people were evaluated, and the correlation between neurological headache, and anxiety and depression were analyzed. Results: In the control group, the number of people with mild, moderate, and severe anxiety were three, two, and zero, accounting for $3.30 \%, 2.20 \%$, and $0.00 \%$, respectively. The total anxiety was $5.50 \%$, and the SAS score was $31.23 \pm 3.22$; In the observation group, the number of people with mild, moderate, and severe anxiety were fourteen, eleven, and six, accounting for $15.73 \%, 12.36 \%$, and $6.74 \%$, respectively. The total anxiety was $34.83 \%$, and the SAS score was $49.56 \pm 4.98$, which was measured. The data shows that the control group is significantly lower than the observation group in terms of the number of people or the anxiety score, and both $\mathrm{P}<0.05$ are statistically significant; the number of mild, moderate, and severe depression in the control group is four, two, and zero, respectively. They were $4.40 \%, 2.20 \%, 0.00 \%$, the total depression was $6.60 \%$, and the SAS score was $33.23 \pm 3.24$; the number of people with mild, moderate, and severe depression in the observation group were sixteen, eleven, and five, respectively. $17.98 \%, 12.36 \%$, $5.62 \%$, total depression degree was $35.96 \%$, SAS score was $46.56 \pm 4.68$. The measurement data showed that the control group was significantly lower than the observation group in terms of the number of people or the depression score, and both were $\mathrm{P}<0.05$, which was statistically significant. Conclusion: Patients with neuropathic pain are usually accompanied by anxiety and depression. Anxiety and depression are also important factors that affect patients with neuropathic pain. Psychotherapy intervention for patients is very necessary to relieve patients' anxiety and depression. Thereby reducing the pain of the patient.
\end{abstract}

Keywords: Neuropathic headache; Depression and anxiety; Correlation analysis

Publication date: July 2021; Online publication: July 31, 2021

\section{Introduction}

With the increase in life pressure and changes in daily life habits, the number of people with neuropathic headaches are increasing year by year. When neuropathic headaches occur, the symptoms are usually persistent headaches, and straining nerves in the brain. A strong sense of oppression and too much pain will lead to changes in the patient's mood, constantly increasing the number of negative emotions, and even severe anxiety and depression. Patients with neuropathic headaches are usually accompanied by some complications. Occurrence, such as memory loss, emotional instability, loss of interest in life, etc., these symptoms will cause the patient's neuropathic headache treatment to be prolonged, and the curability is reduced, also the prognostic effect is not obvious. Therefore, this article studies neuropathic headache and the relevance of anxiety and depression, which provides data support for psychological intervention in 
clinical medicine, is now reported as follows.

\section{Materials and methods}

\subsection{General information}

From June 2019 to May 2021, a total of 89 neuropathic headache patients were treated in our hospital and they were recorded as the observation group. Healthy people in the same period used random number table method to randomly screen 91 cases and recorded as the control group. The observation was approved by the ethics committee, and the patient had known the situation and had obtained the consent of a healthy person. Exclude patients with other co-infections, headaches caused by other factors, and patients during lactation and pregnancy, and exclude patients with chronic diseases; patients with neuropathic headache between 20-65 years old will be automatically withdrawn or after testing, the headache lasts for 30 minutes/day, more than seven days.

\subsection{Method}

The control group used questionnaire surveys and used self-rating anxiety scale (SAS) and selfrating depression scale (SDS) scores to evaluate the anxiety and depression of healthy people. The observation group also used SAS and SDS scores before admission to the hospital to evaluate the anxiety and depression of neuropathic headache patients. In addition, a visual analog scale (VAS) assessment is required. First, the staff needs to be trained, the content and questions of the questionnaire should be formulated, and the questionnaire should be formulated according to the international SAS and SDS scoring table. The staff should guide the patient in the filling process. Then, after completing the filling, the questionnaires need to be collected in groups, and VAS assessment is carried out to analyze the correlation between neurological headache and anxiety and depression.

\subsection{Observation index and effect judgment}

(1) Anxiety is measured using the internationally-used anxiety self-evaluation scale. The scale contains 20 measurement items with a total of 80 points. Each item has four levels, each with a score of 1-4 and a score of less than 50. Yes, it means that there is no anxiety. Those with a score of 50-59 indicate mild anxiety; those with a score of 60-69 indicate moderate anxiety; those with a score greater than 69 indicate severe anxiety.

(2) Depression is also measured using the depression self-evaluation scale. The scale contains a total of 20 measurement items, a total of 80 points, and each item has four levels, respectively 1-4 points, with a score less than 53, Means no depression, those with a score of 53-62 indicate mild depression; those with a score of 63-72 indicate moderate depression; those with a score greater than 72 indicate severe depression.

(3) The degree of pain is evaluated by VAS, and the total score is 10 points. The patient points out the position that can represent his pain degree according to his own pain degree, and the doctor gives a score according to the position marked by the patient. Among them, 0 point means no pain. A score of 3 or less indicates that there is tolerable slight pain; a score of 4 to 6 indicates that the pain is still tolerable, which will affect the quality of sleep; a score of 7-10 indicates that the pain is unbearable, which not only affects the quality of sleep, but also affects the quality of sleep the patient's appetite.

\subsection{Statistical methods}

SPSS 20.0 statistical software was used for data analysis. The count data was represented by [n(\%)], and the comparison was performed by the $\mathrm{X}^{2}$ test. The SAS, SDS, and VAS scores were represented by the $\mathrm{t}$ test, which was represented by $\overline{\mathrm{x}} \pm \mathrm{s}$, and $\mathrm{P}<0.05$ was considered statistically significant. 


\section{Results}

\subsection{Comparison of SAS scores and anxiety of the two groups of patients}

The anxiety of the two groups of patients was compared. The number of people with mild, moderate, and severe anxiety in the control group were three, two, and zero, respectively, accounting for $3.30 \%, 2.20 \%$, and $0.00 \%$, respectively, and the total anxiety was $5.50 \%$. The SAS score was $31.23 \pm 3.22$. The number of people with mild, moderate, and severe anxiety in the observation group was fourteen, eleven, and six, accounting for $15.73 \%, 12.36 \%$, and $6.74 \%$, respectively. The total anxiety was $34.83 \%$, and the SAS score It was $49.56 \pm 4.98$. The measured data showed that the control group was significantly lower than the observation group in terms of the number of people or the anxiety score, and both were $\mathrm{P}<0.05$, which was statistically significant.

Table 1. Comparison of SAS scores and anxiety between the two groups.

\begin{tabular}{cccccc}
\hline \multirow{2}{*}{ Group } & \multirow{2}{*}{ SAS score } & \multicolumn{2}{c}{ Anxiety positive [n(\%)] } & Anxiety [n(\%)] \\
\cline { 3 - 5 } & & Mild & Moderate & Severe & \\
\hline Control group (n=91) & $31.23 \pm 3.22$ & $3(3.30)$ & $2(2.20)$ & 0 & $5(5.50)$ \\
Observation group (n=89) & $49.56 \pm 4.98$ & $14(15.73)$ & $11(12.36)$ & $6(6.74)$ & $31(34.83)$ \\
$\mathrm{t} / \mathrm{X}^{2}$ & 29.3888 & & & \\
$\mathrm{P}$ & 0.0000 & & & 0.000 \\
\hline
\end{tabular}

\subsection{Comparison of SDS scores between the two groups}

The depression of the two groups of patients was compared. The number of mild, moderate, and severe depression in the control group were four, two, and zero, accounting for $4.40 \%, 2.20 \%$, and $0.00 \%$, respectively, and the total depression was $6.60 \%$. The SAS score was $33.23 \pm 3.24$. The number of people with mild, moderate, and severe depression in the observation group were 16, 11, and five, accounting for $17.98 \%, 12.36 \%$, and $5.62 \%$, respectively. The total and depression degree was $35.96 \%$. SAS The score was $46.56 \pm 4.68$. The measurement data showed that the control group was significantly lower than the observation group in terms of the number of people or the depression score, and both were $\mathrm{P}<0.05$, which was statistically significant.

Table 2. Comparison of SDS scores and conditions between the two groups

\begin{tabular}{cccccc}
\hline \multirow{2}{*}{ Group } & \multirow{2}{*}{ SDS score } & \multicolumn{3}{c}{ Depression positive [n(\%)] } & Depression \\
\cline { 3 - 5 } & & Mild & Moderate & Severe & {$[\mathbf{n}(\%)]$} \\
\hline Control group $(\mathrm{n}=91)$ & $33.23 \pm 3.24$ & $4(4.40)$ & $2(2.20)$ & 0 & $6(6.60)$ \\
Observation group $(\mathrm{n}=89)$ & $46.56 \pm 4.68$ & $16(17.98)$ & $11(12.36)$ & $5(5.62)$ & $32(35.96)$ \\
$\mathrm{t} / \mathrm{X}^{2}$ & 27.2690 & & & 23.2913 \\
$\mathrm{P}$ & 0.0000 & & & 0.0000 \\
\hline
\end{tabular}




\section{Disscussion}

Neuropathic headache is a common disease in neurology. Although the clinical prevalence of the disease is high, more patients are less aware of it, also more patients have a longer duration of disease, which seriously affects the quality of life of patients. In addition, patients in this category experience negative emotions due to pain and other discomforts. Anxiety and depression are prominent aspects. Some studies believe that the existence of these negative emotions can lead to long-term illness or even worsening, but there is still a lack of research. The relationship between headache severity and anxiety and depression scores leads to lack of reference for the formulation of disease control measures. In addition, although there are many studies on negative emotions that affect the onset of neuropathic headache patients in existing clinical studies, there are still gaps in related studies, which further increase the demand and importance of clinical research.

The more serious the depression and anxiety, the more obvious the expression of neurotransmitters, the greater the impact on the body, the significant changes in neuromuscular pressure, and the worsening of pain perception. Emotion control is one of the key points of clinical intervention, and the purpose of pain control can be achieved by regulating negative emotions.

To sum up, patients with neuropathic pain are usually accompanied by anxiety and depression. Anxiety and depression are also important factors that affect patients with neuropathic pain. It is very necessary to provide psychological interventions for patients to relieve anxiety and depression of patients. Emotions, thereby alleviating the pain of the patient.

\section{Disclosure statement}

The author declares no conflict of interest.

\section{References}

[1] Liu XM, 2019, What are the Causes of Headaches?, Wenhui of Health Care, (10):272.

[2] Chi ZL, Guo JH, 2019, Clinical Observation of Headache Symptoms in Neurology. Digest of World Latest Medical Information, 19(57): 109-110.

[3] Feng SX, 2019, Research on the Etiology and Treatment of Neuropathic Headache. Digest of World Latest Medical Information, 19(53): 101+103.

[4] Chen YM, 2019, The Promotion of Health Education in the Rehabilitation of Patients with Neuropathic Headache. Capital Food and Medicine, 26(11): 171.

[5] Liu L, 2019, Clinical Observation and Analysis of Headache Symptoms in Neurology. Electronic Journal of Clinical Medicine Literature, 6(40): 40. 